Apidologie, 1976, 7 (4) 307-330.

\title{
NOUVELLES OBSERVATIONS SUR L'ÉLEVAGE DES MÂLES DANS LES COLONIES D'ABEILLES (APIS MELLIFICA L.)
}

\author{
Neue Beobachtungen über die Drohnenaufzucht \\ in Bienenvölkern (Apis mellifica L.)
}

\section{J. MESQUIDA}

Laboratoire de Recherches de la Chaire de Zoologie, E.N.S.A., I.N.R.A.

65, rue de St-Brieuc, 35042 Rennes Cedex

\author{
SUMMARY \\ FURTHER OBSERVATIONS ON DRONE REARING IN HONEYBEE COLONIES \\ (Apis mellifica L.)
}

The author gives the results of standardized observations in 1972 and 1973 upon honeybees colonies (Apis mellifica L.) which present the character (c reduced or null male breeding 》 with the purpose to attempt the study of the origin of the mechanism of the phenomenon.

It has been established that this phenomenon does not seem to proceed from a failure in the cell size evaluation by the queen as it does not seem to proceed neither of a failure in the honeycomb building nor of the brood destruction by workers. This phenomenon seems to compare itself more with the GoRBAKov hypothesis and would be concerned with the working of the spermatheca. But a verification of this hypothesis with a greater number of colonies with the character « without male 》 is yet necessary before a conclusion can be drawn. The absence or the reduction of drone brood is nevertheless an hereditary character which seems to be submitted to the influence of many gena.

\section{RÉSUMÉ}

L'auteur donne les résultats d'une série d'observations effectuées en 1972 et 1973 sur des colonies d'abeilles (Apis mellifica L.) présentant le caractère c élevage de mâles réduit ou nul » dans le but de tenter l'étude de l'origine et du mécanisme du phénomène.

Il a été établi que ce phénomène ne semble pas relever d'une défaillance de l'évaluation de la taille des cellules par les reines de même qu'il ne semble pas relever du défaut de construction de rayons ni de la destruction du couvain par les ouvrières. Il semble se rapprocher davantage 
de l'hypothèse de Gorbakov et concernerait alors le fonctionnement de la spermathèque. Mais une vérification de cette hypothèse sur un plus grand nombre de colonies à caractère " élevage sans mâles " serait nécessaire. L'absence ou la réduction de production de couvain de mâles est en tout cas un caractère héréditaire qui semble soumis à l'influence de plusieurs gènes.

\section{I. - INTRODUCTION}

Dans des conditions normales, en dehors de toute influence des techniques apicoles, les colonies d'abeilles élèvent toujours une certaine quantité de mâles pendant une courte période de l'année. La proportion du couvain mâle par rapport au couvain total produit est très variable selon les colonies (M. D. Allen, 1958). Ainsi chez l'abeille carniolienne (A.m. carnica Pollmann) Weiss $(1962,1969)$ indique des proportions de surfaces de couvain de mâles qui varient de 7,5 à $18,7 \%$ au moment du maximum de production. Chez l'abeille noire française ( $A$. m. mellifica L.) nous avons nous-mêmes noté en 1970 et en 1971 une variation de 5 à $14 \%$ (Louveaux, Mesquida et Fresnaye, 1972).

En faisant intervenir des techniques apicoles particulières il est possible d'obtenir d'importantes modifications de cette production normale de couvain de mâles. M. D. Allex $(1963,1965)$ a montré qu'on peut la réduire par destruction de cellules de mâles ou, au contraire, l'augmenter considérablement par introduction de cadres de cellules de mâles. Dans l'un et l'autre cas elle constate aussi une grande variabilité de production de couvain de mâles entre les colonies. Notons que l'introduction de cadres de cellules de mâles, bâtis ou non, est une opération courante connue depuis longtemps des apiculteurs éleveurs de reines : ils l'emploient dans le but d'augmenter les populations de mâles de leur région afin d'assurer une meilleure fécondation.

Koeniger (1970) a montré l'existence d'un rapport entre le mécanisme de fécondation de l'œuf et le type de cellule dans laquelle cet œuf a été pondu. Il aboutit à la conclusion que la reine évalue d'abord la taille de la cellule à l'aide de ses pattes antérieures et qu'elle pond ensuite un ouf fécondé ou non selon que la cellule est de petite ou de grande dimension. Expérimentalement, par amputation des pattes antérieures jusqu'au trochanter, cet auteur a montré la possibilité de conditionner la capacité de production de mâles d'une reine d'abeilles.

Mais les modifications de production de couvain de mâles les plus importantes en amplitude sont celles qui présentent le caractère d'une anomalie. La production de mâles peut être alors c nulle ) ou très c réduite " par rapport aux colonies normales. Différents cas ont été signalés ou étudiés par Michailov (1927), Gorbakov (1961), Drescher (1968).

Dans les exemples cités par Michailov et Gorbakov, il s'agit de reines incapables de pondre des eufs de mâles. D'après Gorвakov, ce phénomène 
serait provoqué par une défaillance du mécanisme obturateur de la spermathèque dépendant de l'état homozygote d'un gène récessif.

Dans des colonies fortement consanguines et en liaison avec un manque de vitalité, Drescher (1968) a constaté fréquemment une tendance à une production de couvain de mâles peu importante.

Nous avons nous-mêmes observé des cas de production très faible de mâles dans des colonies hybrides dont l'une des souches parentales provenait d'un élevage consanguin (Louveaux, Mesquida, Fresnaye, 1972). Il nous a semblé que la consanguinité avait constitué un facteur favorisant l'apparition de ces colonies élevant très peu de mâles. Cet élevage de mâles réduit, qui s'est trouvé par la suite peu modifié d'une génération à l'autre lorsque des croisements successifs ont fait disparaître la consanguinité, a permis de montrer le caractère héréditaire du phénomène observé. Ces premières études, encore insuffisantes pour donner une explication valable du mécanisme du phénomène, nous ont conduit néanmoins à retenir trois hypothèses de travail.

La première concerne la reine. Si la reine est à l'origine du phénomène, il pourrait s'agir, soit d'une défaillance dans l'évaluation de la taille des cellules, soit d'une défaillance dans le fonctionnement de la spermathèque. L'absence de ponte dans les grandes cellules pourrait signifier une bonne évaluation de la taille des cellules (Koeniger, 1970) et correspondre à un mauvais fonctionnement de la spermathèque (hypothèse de Gorbakov). Inversement, on pourrait concevoir que la présence d'œufs donnant des mâles dans les petites cellules et d'œufs donnant des ouvrières dans les grandes cellules pourrait signifier un bon fonctionnement de la spermathèque de la reine, mais l'erreur pourrait être due alors à l'évaluation défectueuse de la taille des cellules. Le fonctionnement de la spermathèque serait aussi en cause si les cufs pondus dans les petites et les grandes cellules donnaient uniquement des ouvrières. La mauvaise évaluation de la taille des cellules pourrait être d'origine accidentelle comme il pourrait aussi s'agir d'un comportement anormal ou même d'une malformation des pattes antérieures. Dans le cas du mauvais fonctionnement de la spermathèque il s'agirait surtout d'une malformation congénitale ou d'une anomalie de comportement d'origine génétique.

La seconde et la troisième hypothèses concernent les ouvrières. En effet, si la reine n'est pas à l'origine du phénomène ce sont les ouvrières qui le seraient. Il pourrait s'agir soit d'une incapacité à construire des cellules de mâles soit d'une destruction systématique du couvain de mâles normal à l'état d'œuf comme Woyke $(1963,1965,1972)$ l'a observée pour les mâles diploïdes.

Étant donné l'intérêt apicole du problème nous avons mis en place au cours de ces dernières années une série d'observations qui avaient pour objet de vérifier ces hypothèses et de tenter l'étude de l'origine et du mécanisme du phénomène. 


\title{
II. - MATÉRIEL ET MÉTHODE
}

\author{
A. - Les colonies d'abeilles
}

Les observations ont été effectuées en 1972 et 1973 dans un rucher situé au Rheu près de Rennes (Ille-et-Vilaine) en Bretagne.

Les ruches utilisées étaient du modèle Dadant-Blatt 10 cadres.

Les observations ont porté sur trois lots de colonies d'abeilles constitués en fonction de leur production de couvain de mâles.

Lot I. - Ce lot n'a pu être constitué en 1972 et 1973 qu'avec une seule colonie d'abeilles (No 32) dont la production de couvain de mâles a été nulle. La reine était arrière-petite-fille de la reine d'origine (4e génération).

Lot II. - Ce lot a été constitué avec quatre colonies ( $N^{\circ} 15,53,43$ et 3 ) dans lesquelles les reines ont toujours produit une certaine quantité de couvain de mâles. En 1972 les reines de ces colonies étaient toutes petites-filles de la reine d'origine (3e génération) et en 1973 arrièrepetites-filles ( $4^{\mathrm{e}}$ génération) de la reine d'origine.

Ces deux premiers lots faisaient partie à l'origine du groupe de quatre colonies hybrides de 1967 (No 15, 32, 22 et 13) et du groupe de cinq colonies hybrides de 1968 (No 53, 65, 43, 58 et 3) (Louveaux, Mesquima, Fresnaye, 1972) dont quatre (No 22, 13, 65 et 58) ont été perdues pendant l'hivernage 1971-1972. Il s'agissait de deux lots de reines sceurs obtenus, l'un en 1967 et l'autre en 1968, par élevage à la Station Expérimentale d'Apiculture de l'I.N.R.A. à Montfavet (Vaucluse). Du côté maternel, ces reines descendaient d'une souche italienne non consanguine, de race pure ( $A$. m. ligustica), importée d'Italie en 1965 ; leur mère avait été inséminée artificiellement avec le sperme de mâles de race noire $(A . m$. mellifica) appartenant à une souche provençale (No 4.15 en 1967 et No 360 en 1968) conservée depuis longtemps en étroite consanguinité par insémination artificielle. Les reines de première génération reçues en 1967 et 1968 avaient été fécondées en Provence par les mâles locaux qui sont de race noire dans leur très grande majorité. On peut donc considérer que ces reines étaient des reines hybrides de races présentant un génotype relativement homogène.

Dans ces deux lots on a laissé les reines des générations successives se renouveler librement. On s'est contenté de noter les changements de générations.

Lot III. - Ce lot a été constitué chaque année de colonies d'abeilles avec reines de la race noire (A. m. mellifica) appartenant à une population bretonne locale dont la production de couvain de mâles est toujours apparue normale (colonies No 2,37 et 55 en 1972 et No $8,47,70$ et 68 en 1973). Ces colonies ont constitué les témoins locaux et celles qui ont renouvelé spontanément leur reine ont été éliminées et remplacées par d'autres ayant une reine de la souche bretonne.

\section{B. - Les mesures des surfaces de couvain}

Les mesures des surfaces de couvain total ont été effectuées par la méthode de Fressaye (1962) et les mesures des surfaces de couvain de mâles par la méthode décrite en 1972 (Louveaux, Mesquida, Fresnaye). En 1972 ces mesures ont été effectuées en une seule fois, le 25 juin, au moment du maximum de production du couvain. En 1973 elles ont été faites mensuellement du mois de mars au mois de septembre.

\section{C. - Les rendements en miel}

Les rendements en miel ont été calculés en tenant compte des nourrissements effectués et des poids des ruches après les nourrissements d'automne, au moyen de la formule :

$$
\mathbf{R}=\mathbf{M}+(\mathbf{H} 2-\mathbf{H} \mathbf{1})-\mathbf{N}
$$

où $\mathrm{R}=$ rendement $\mathrm{M}=$ miel extrait des ruches; $\mathrm{H} 2=$ Poids de la ruche à la fin de l'année de l'expérience après les nourrissements d'automne; $H 1=$ poids de la ruche au début de l'expérience après les nourrissements d'automne; $N=$ poids du sucre donné en nourrissement (LAvIE, 1968). 


\section{D. - Le calcul statistique}

L'interprétation statistique des résultats a été effectuée par comparaisons de moyennes : test $t$ de STUDENT pour les valeurs conformes aux conditions d'application du test ou test non paramétrique de Kruskall ex W Allis dans le cas contraire. Pour les poureentages, le test a été appliqué après transformations en valeur angulaire où $y=2$ are Sin $\sqrt{x}$ (DAGNELIE, 1969, 1970).

\section{E. - La conduite des ruches}

En 1972, on n'a procédé à aucune opération susceptible d'augmenter la ponte ou d'inciter la construction cirière des colonies.

Pour les besoins des observations, en 1973 la production de couvain de mâles a été au contraire poussée au maximum dans toutes les colonies des lots I à III. La méthode a consisté à introduire ad libitum dans les colonies autant de cadres de cire gaufrée à cellules de mâles que la reine est susceptible d'en garnir successivement de ponte entre le mois de mars et le mois de juillet. Dans chaque ruche le cadre de cire gaufrée à cellules de mâles a toujours été introduit au bord du nid à couvain. On a procédé à l'introduction d'un seul cadre à la fois et on a attendu qu'il soit bâti et entièrement garni de couvain pour introduire un autre cadre. Les feuilles de cire gaufrée à cellules de mâles utilisées étaient de $41 \times 20 \mathrm{~cm}$ et à 510 cellules au décimètre carré. Les colonies d'abeilles ont toutes été nourries au sirop de sucre dilué à raison de $250 \mathrm{ml}$ par ruche et par semaine du 8 mars au 10 avril.

\section{F. - Les constructions cirières sur “ cadres têmoins 》}

L'aptitude des ouvrières à la construction des cellules de mâles a été vérifiée par utilisation de la méthode dite du " cadre témoin ». Elle a consisté à introduire un cadre vide dépourvu de cire appelé " cadre témoin " de dimension normale $43,5 \times 30 \mathrm{~cm}$ au bord du nid à couvain de chaque ruche. Chaque semaine ce " cadre témoin " était retiré de la ruche avec ses constructions cirières et aussitôt remplacé au même endroit dans la ruche par un nouveau " cadre témoin ".

Les contours des constructions cirières hebdomadaires tant en cellules de mâles qu'en cellules d'ouvrières étaient dessinés par transparence sur des feuilles rigides de chlorure de polyvinyle et les surfaces étaient mesurées au laboratoire à l'aide d'un planimètre.

Cette technique est inspirée des travaux de Paschre (1939); elle est aussi appelée " Technique du cadre témoin "par Brisset (1946). C'est une technique couramment employée par les apiculteurs allemands sous le nom de “ Baurahmen » (HeroLD, 1960).

\section{G. - Le taux de destruction du couvain de mâles}

Pour l'évaluation du taux de destruction du couvain de mâles par les ouvrières la méthode utilisée se rapproche de celle décrite par MAUL (1972) pour l'étude du couvain lacunaire. Elle consiste à introduire entre les deux premiers cadres du nid à couvain de la ruche un cadre entièrement bâti en cellules de mâles. Quatre jours après son introduction, lorsqu'il est garni d'œufs, un repère en matière plastique est fixé au rayon. On dénombre ensuite à différents stades de développement le couvain compris à l'intérieur de ce repère depuis le stade cuf jusqu'à quelques jours de l'éclosion. Le repère est constitué d'un cadre en matière plastique ayant la forme d'un parallélogramme de $16 \times 8 \mathrm{~cm}$ et renfermant environ 250 cellules de mâles. La fixation au rayon est assurée par des épingles soudées sur les côtés de ce cadre.

\section{H. - Description des schémas expérimentaux}

1) Étude du phénomène dans l'hypothèse où il serait lié au comportement de la reine.

Dans le but de vérifier si l'absence ou la réduction de l'élevage du couvain de mâles dépend de la reine ( $1^{\text {re }}$ hypothèse) les observations ont été effectuées régulièrement deux fois par semaine du mois de mars au mois de juillet 1973. Elles ont porté sur toutes les colonies des lots I à III. Elles ont consisté à examiner à chaque fois et dans chaque ruche tous les cadres du nid à couvain. On s'est contenté de noter la présence du couvain dans les cellules de grande taille situées dans les angles inférieurs des vieux rayons ou sur ceux nouvellement bâtis. Il s'agissait de vérifier aussi si c'était bien du couvain de mâles. 
2) Étude du phénomène dans l'hypothèse où il serait liê au comportement des ouvrières.

a) par l'intermédiaire des constructions cirières

Pour vérifier si l'absence ou la réduction de l'élevage du couvain de mâles dépend du comportement des ouvrières incapables de construire des cellules de mâles ( $2^{\mathrm{e}}$ hypothèse) deux séries d'essais ont été effectuées en 1973 :

- l'une en présence de cadres de cire gaufrée à cellules de mâles,

- l'autre sur « cadres témoins ».

La première effectuée en mars et en avril a porté sur l'ensemble des colonies des lots I à III. Dans chacune d'elles un cadre de cire gaufrée à cellules de mâles a été introduit au bord du nid à couvain, entre celui-ci et un cadre de réserve.

La série d'essais utilisant la méthode des constructions cirières sur "cadre témoin " décrite précédemment a été effectuée du 15 juin au 19 juillet pendant la période de la miellée. Elle a porté également sur toutes les colonies des lots I à III et les comparaisons ont été faites sur les surfaces totales des constructions cirières, sur les surfaces des constructions en cellules de mâles et sur celles en cellules d'ouvrières.

b) par l'intermédiaire de la destruction du couvain

En vue de vérifier si l'absence ou la réduction de production de couvain de mâles dépend de la destruction du couvain de mâles par les ouvrières ( $3^{\mathrm{e}}$ hypothèse) la méthode décrite précédemment a porté sur trois colonies du lot II (No 15,3 et 43 ) et les quatre colonies du lot III. Les essais ont été effectués le 10 avril, le 2 mai et le 26 juin 1973.

\section{III. - RÉSULTATS}

\section{A. - Production de couvain de mâles dans la descendance des différents lots}

En 1972 et 1973 les mesures de surfaces de couvain, groupées dans les tableaux 1 et 2, font apparaïtre de nouveau une importante variabilité de la production de couvain de mâles dans la descendance des colonies des lots I et II. C'est ainsi que l'on constate que la colonie $N^{\circ} 32$ (lot I) a conservé le caractère d'origine pendant les deux années consécutives.

Par contre les colonies du lot II (No 15, 53, 43 et 3) ont fourni un peu plus de couvain de mâles que les années antérieures. Les différences étant non significatives dès 1972 , on constate une tendance à une production normale, bien qu'elle soit restée encore inférieure à celle dés colonies bretonnes.

En 1973, le tableau 2 fait apparaître des surfaces de couvain de mâles nettement plus élevées qu'en 1972 en raison de l'application systématique de la méthode d'élevage de mâles poussée au maximum. Mais, malgré cela, les surfaces moyennes de couvain de mâles des colonies du lot II restent inférieures à celles des bretonnes (lot III). Ces différences ne sont pas significatives. C'est la colonie bretonne $\mathrm{N}^{\circ} 47$ qui a fourni les plus grandes surfaces de couvain de mâles en fin juin : $3800 \mathrm{~cm}^{2}$ pour une surface de couvain total de $11000 \mathrm{~cm}^{2}$, soit $32 \%$. 
TABL. 1. - Surfaces de couvain (ouvrières et mâles) observées dans les lots 1 à III au Rheu le 28 juin 1972 TAB. 1. - Brutflächen (Arbeiterinnen u. Drohnen) in den Gruppen I bis III (Rheu bei Rennes) am 28.6.1972

\begin{tabular}{|c|c|c|c|c|c|}
\hline \multirow{2}{*}{$\begin{array}{c}\text { Lots } \\
\text { Gruppen }\end{array}$} & \multirow{2}{*}{$\begin{array}{l}\text { No Ruche } \\
\text { Nr. d. } \\
\text { Volks }\end{array}$} & \multirow{2}{*}{$\begin{array}{l}\text { Génération } \\
\text { Generation }\end{array}$} & \multicolumn{3}{|c|}{$\begin{array}{c}\text { Surfaces de Couvain } \\
\text { Brutfächen }\end{array}$} \\
\hline & & & en $\mathrm{cm}^{2}$ & $\partial \mathrm{en} \mathrm{cm}^{2}$ & o en $\%$ \\
\hline$I^{1}$ & 32 & 4 & 6.160 & 0 & 0 \\
\hline II & $\begin{array}{l}15 \\
43 \\
03\end{array}$ & $\begin{array}{r}3 \\
2-3 \\
3\end{array}$ & $\begin{array}{l}9.598 \\
5.925 \\
7.618\end{array}$ & $\begin{array}{r}1.322 \\
195 \\
862\end{array}$ & $\begin{array}{r}12 \\
3 \\
10\end{array}$ \\
\hline $\begin{array}{l}\text { Moyennes } \varnothing \quad \ldots \ldots \ldots \ldots \ldots \ldots \\
\text { Écarts-types } \\
\text { Abweichungen }\end{array}$ & & & $\begin{array}{r}7.713 \\
( \pm 1.838)\end{array}$ & $\begin{array}{r}793 \\
( \pm 567)\end{array}$ & $\begin{array}{r}8,3 \\
( \pm 4,7)\end{array}$ \\
\hline $\begin{array}{l}\text { III Bretagne (Témoin) } \ldots \ldots \ldots \\
\text { (Kon trolle) }\end{array}$ & $\begin{array}{l}02 \\
37 \\
55\end{array}$ & $\begin{array}{l}1 \\
1 \\
1\end{array}$ & $\begin{array}{r}8.594 \\
10.686 \\
8.060\end{array}$ & $\begin{array}{l}1.446 \\
1.554 \\
1.060\end{array}$ & $\begin{array}{l}14 \\
13 \\
11\end{array}$ \\
\hline $\begin{array}{l}\text { Moyennes } \varnothing \ldots \ldots \ldots \ldots \ldots \ldots \\
\text { Écarts-types } \\
\text { Abweichungen }\end{array}$ & & & $\begin{array}{r}9.113 \\
( \pm 1.383)\end{array}$ & $\begin{array}{r}1.353 \\
( \pm 260)\end{array}$ & $\begin{array}{r}12,3 \\
( \pm 1,5)\end{array}$ \\
\hline $\begin{array}{l}\text { Différences entre lots II et III ... } \\
\text { Unterschiede zw. Gruppe II u. III }\end{array}$ & & & NS & NS & NS \\
\hline
\end{tabular}

1. le lot I n'a pu ètre constitué en 1972 et 1973 qu'avec une seule colonie.

Gruppe I bestand 1972 u. 1973 nur aus einem einzigen Volk.

* indique que la moyenne est significativement différente de celle du groupe d'abeilles de Bretagne à $\mathrm{P}=0,10$ (test $\mathrm{t})$.

zeigt an, dass der Durchschnitt sich deutlich von dem der bretonischen Völker unterscheidet; $P=0,10$ (t-Test).

** = différence significative à $\mathbf{P}=\mathbf{0 , 0 5}$

deutlicher Unterschied; $P=0,05$

*** =: différence significative à $\mathbf{P}=0,01$

deulicher Unterschied; $P=0,01$

NS :- différence non significative

kein wesentlicher Unterschied

- En ce qui concerne les générations la convention suivante a été adoptée :

$1=$ reine d'origine $\quad 3=$ reine petite-fille de la reine d'origine

2 = reine fille de la reine d'origine $\quad 4=$ reine arrière-petite-fille de la reine d'origine

- In bezug auf die Generationen gilt. folgendes :

$1=$ Ausgangskönigin $\quad 3=$ Enkelin d. 1. Königin

$2=$ Tochter d. Ausgangskönigin $\quad 4=$ Urenkelin d. 1. Königin

- Les proportions de couvain de mâles sont données en \% du couvain total.

- Die Proportionen der Drohnenbrut sind in \% der Gesamtbrut angegeben. 


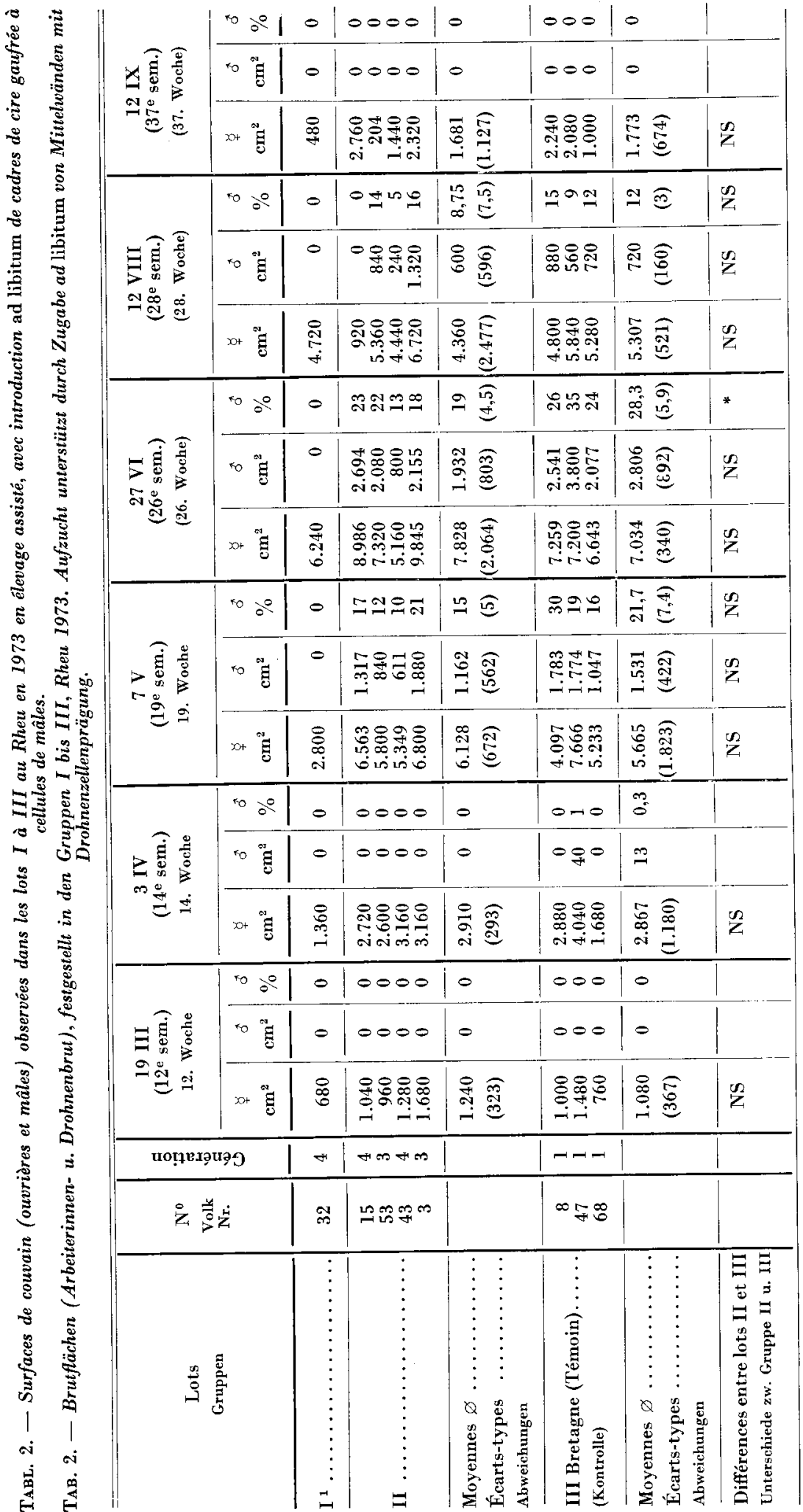

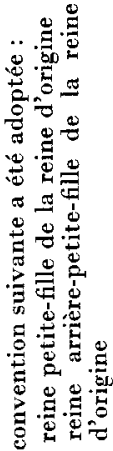




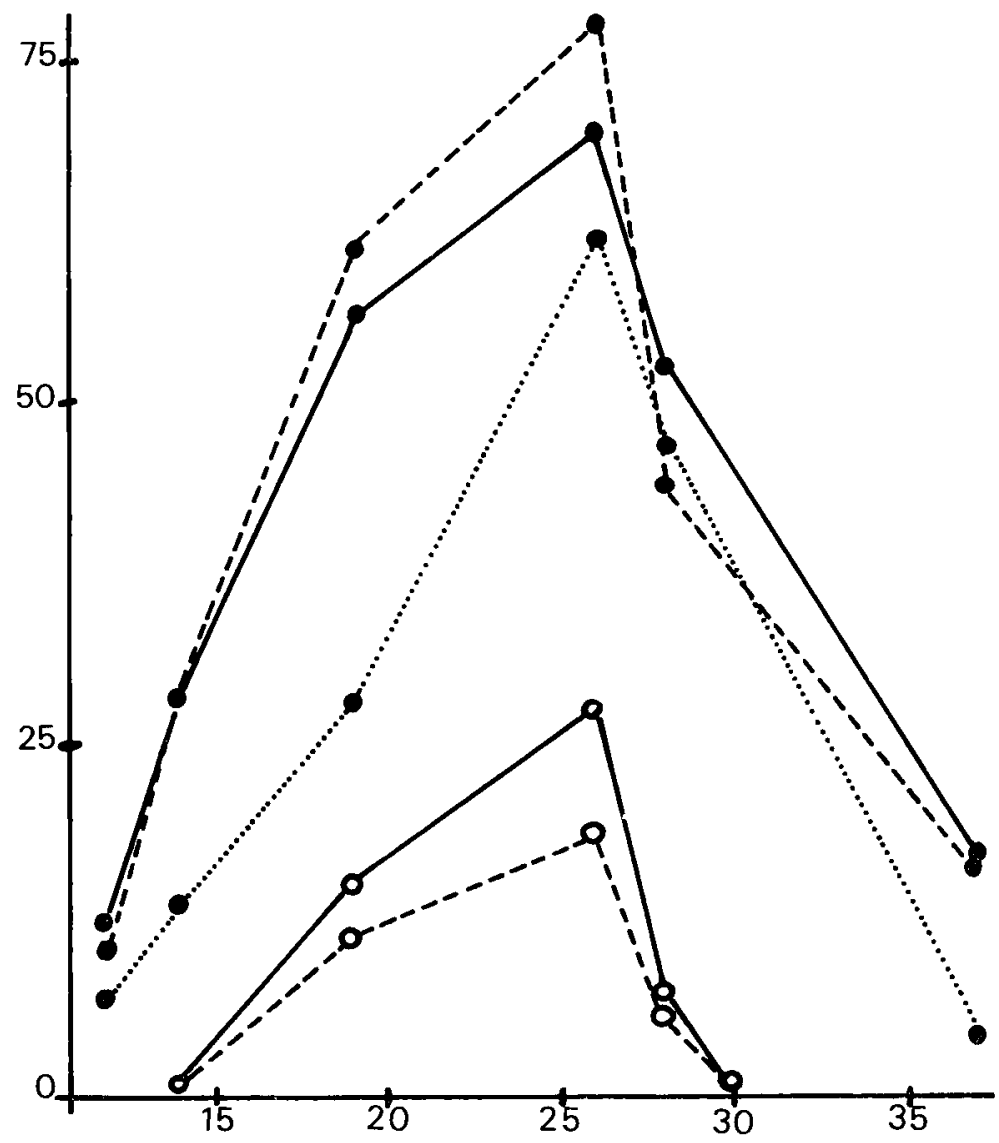

FIG. 1. - Surfaces moyennes mensuelles de la production de couvain total et de couvain de mâles des colonies d'abeilles des lots I à III en 1973.

Ordonnées : surfaces en $\mathrm{dm}^{2}$.

Abscisses : semaines

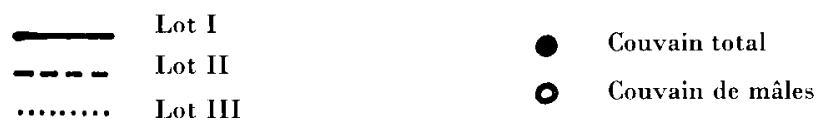

Aв8. 1. - Monatliche durchschnitliche Brutflächen - Gesamtbrut und Drohnenbrut in den Bienenvölkern der Gruppen I-III, 1973 .

Ordinate $=$ Brutfläche in $\mathrm{cm}^{2}$.

Abszisse $=$ Wochen

Gruppe I

$\begin{array}{ll}-\ldots-- & \text { Gruppe II } \\ \ldots . . \ldots \ldots & \text { Gruppe III }\end{array}$
- Gesamtbrutfläche

- Drohnenbrutfläche 


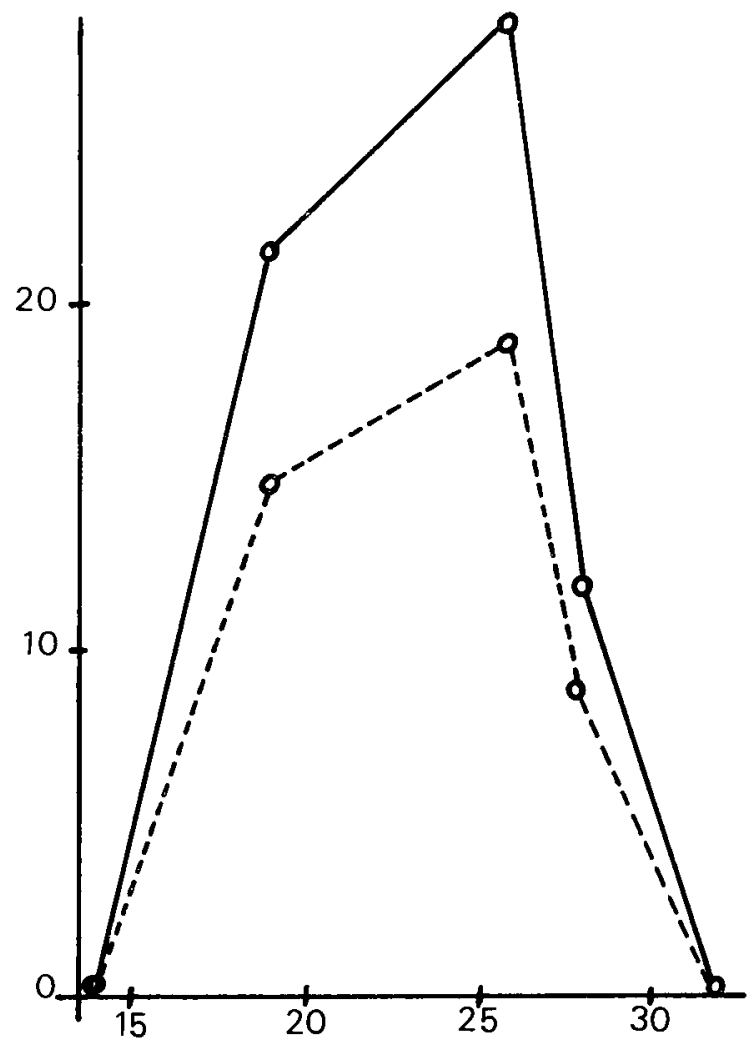

Fig. 2. - Pourcentages moyens mensuels de la production de couvain de mâles des colonies d'abeilles des lots I et II de 1973 à Rennes (données en \% du couvain total).

Ordonnées : couvain de mâles (en \%). Abscisses : semaines.

Lot I

- - - Lot II

Aвв. 2. - Monatlicher durchschnittlicher Prozentsatz der Drohnenbruterzeugung in den Völkern der Gruppen I und II 1973 in Rennes (In \% der Gesamtbrutfüche).

Ordinate $=$ Drohnenbrut (in \%).

Abszisse $=$ Wochen.

Gruppe I

-- -- Gruppe II 
La figure 1 montre que les courbes des surfaces moyennes de couvain total des colonies du lot II et des colonies bretonnes sont identiques. Il n'y a d'ailleurs pas de différences significatives (tableau 2). La courbe de la colonie hybride No 32 (lot I) tend à se déplacer des deux autres; la surface de couvain total a été sensiblement plus faible au printemps.

La figure montre aussi l'existence d'un cycle de production de couvain de mâles qui se situe, en 1973, du mois d'avril (les premières pontes ont été notées le 3 avril) au mois de juillet (les dernières pontes ayant été observées le 10 juillet). La production maximum a eu lieu dans les deux cas en fin juin et correspond au maximum de production de couvain total.

Par contre, les courbes des surfaces moyennes du couvain de mâles (fig. 1) des colonies du lot II et celles des colonies bretonnes ne sont pas rigoureusemeut identiques. Celle des colonies bretonnes prend toujours le dessus mais statistiquement on ne fait pas apparaître de différences significatives.

Des différences significatives peuvent apparaitre, pendant la période de production maximum de couvain (le 27 juin) lorsque l'on compare cette fois les proportions moyennes des deux lots de colonies (tableau 2). La figure 2 montre d'ailleurs que l'amplitude de la différence entre les deux courbes peut être en effet plus grande que celle des courbes de la figure 1 .

Il faut aussi signaler que le rendement moyen en miel des colonies des lots I et II a eu tendance en 1972 à être plus élevé que celui des témoins bien que la récolte moyenne de miel ait été inférieure (tableau 3 ).

En 1973, le rendement n'a pas été aussi bon qu'en 1972 mais il a été presque le même dans tous les lots de colonies. Là aussi, on remarque qu'il y a eu une récolte moyenne de miel supérieure chez les témoins. En ce qui concerne la surproduction de couvain de mâles provoquée en 1973 par introduction maximum de cadres de cire gaufrée à cellules de mâles, il est intéressant de souligner (tableau 4) qu'elle n'a été suivie d'aucun effet défavorable sur la récolte du miel. La figure 3 montre au contraire qu'il y a eu une tendance inverse. La corrélation n'est pas significative mais ce qui est important c'est qu'elle ne soit pas négative $(r=0,65<0,75$ à $\mathrm{P}=5 \%)$.

\section{B. - Influence de la reine sur la variabilité de la production de couvain de mâles}

Les oufs pondus dans les cellules de grande taille des colonies du lot II ainsi que des colonies bretonnes (lot III), ont tous donné normalement naissance à des mâles. L'évaluation de la taille des cellules par les reines de ces colonies est donc apparue correcte. La reine de la colonie No 32 (lot I) s'est cependant comportée d'une toute autre manière. Elle a d'abord refusé de pondre dans les cellules de grande taille pendant une longue période printanière (tableau 2). C'est seulement plus tard, au mois de juin, qu'elle a finalement pondu dans trois 
de ces cellules : il a été obtenu trois ouvrières; les opercules de ces trois cellules étaient d'ailleurs plutôt plats que tombés. L'évaluation de la taille des cellules par la reine n'est donc pas en cause.

ТАвL. 3. - Récoltes et rendements moyens en miel des colonies des lots I à III à Rennes en 1972 et 1973.

TAB. 3. - Honigernten und Durchschnittserträge der Völker in den Gruppen I bis III Rennes 1972 u. 1973.

\begin{tabular}{|c|c|c|c|c|}
\hline \multirow{2}{*}{$\begin{array}{l}\text { Lots } \\
\text { Gruppen }\end{array}$} & \multicolumn{2}{|c|}{1972} & \multicolumn{2}{|c|}{1973} \\
\hline & $\mathbf{M}$ & $\mathbf{R}$ & $\mathbf{M}$ & $\mathbf{R}$ \\
\hline I et II $\ldots \ldots \ldots \ldots$ & $\begin{array}{c}10 \\
( \pm 3,7)\end{array}$ & $\begin{array}{c}6,03 \\
( \pm 2,5)\end{array}$ & $\begin{array}{r}7,2 \\
( \pm 3)\end{array}$ & $\begin{array}{c}0,4 \\
( \pm 1,4)\end{array}$ \\
\hline $\begin{array}{l}\text { III Bretagne (Témoin) } \ldots \ldots \ldots \\
\text { (Kontrolle) }\end{array}$ & $\begin{array}{c}12,5 \\
( \pm 2,1)\end{array}$ & $\begin{array}{l}4,4 \\
( \pm 0,9)\end{array}$ & $\begin{array}{c}10,3 \\
( \pm 1,2)\end{array}$ & $\begin{array}{c}0,6 \\
( \pm 3,3)\end{array}$ \\
\hline $\begin{array}{l}\text { Différences } \\
\text { Unterschiede }\end{array}$ & NS & NS & NS & NS \\
\hline \multicolumn{5}{|c|}{$\begin{aligned} \mathbf{M}= & \text { Miel récolté dans les hausses (en kg). } \\
& \text { Honigernte aus den Honigräumen in kg. }\end{aligned}$} \\
\hline \multicolumn{5}{|l|}{$\mathrm{R}=\underset{\substack{\text { Rendement en miel. (en } \mathrm{kg} \text { ). } \\
\text { Ionigertrag }}}{\text { Ren }}$} \\
\hline \multicolumn{5}{|l|}{ 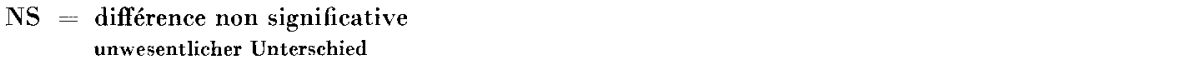 } \\
\hline $\begin{array}{l}\text { Les chiffres entre parenthèses cor } \\
\text { Zahlen in Klammer }=\text { Abweichungen. }\end{array}$ & ndent & types. & & \\
\hline
\end{tabular}

C. - Influence des ouvrières sur la variabilité de production de couvain de mâles

1. - Influence due au comportement de construction.

a) Sur cadres de cire gaufrée à cellules de mâles.

Toutes les colonies, aussi bien la colonie No 32 (lot I) que les colonies du lot II, ont accepté de bâtir des cellules de mâles sur les cadres de cire gaufrée à cellules de mâles introduits en mars et en avril, comme les colonies bretonnes. On a noté seulement quelques variations dans les durées des travaux d'étirage du cadre entier qui n'ont aucun rapport avec l'effet recherché.

b) Sur « cadres témoins 》.

Du 15 au 19 juillet, les données groupées dans le tableau 5 montrent que les colonies des lots I et II et les colonies bretonnes ont construit proportion- 
nellement et globalement presque autant de surfaces de rayons en cellules de mâles qu'en cellules d'ouvrières (entre $53 \%$ et $40 \%$ ).

TABL. 4. - Nombre de cadres de cire gaufrée à cellules de mâles, miel récolté, rendement et production maximum de couvain de mâles des colonies des lots I à III à Rennes en 1973.

TAB. 4. -- Anzahl der Mittelwände mit Drohnenzellenprägung, geernteter Honig, Ertrag und Maximalerzeugung von Drohnenbrut in den Völkern der Gruppen I bis III, Rennes 1973.

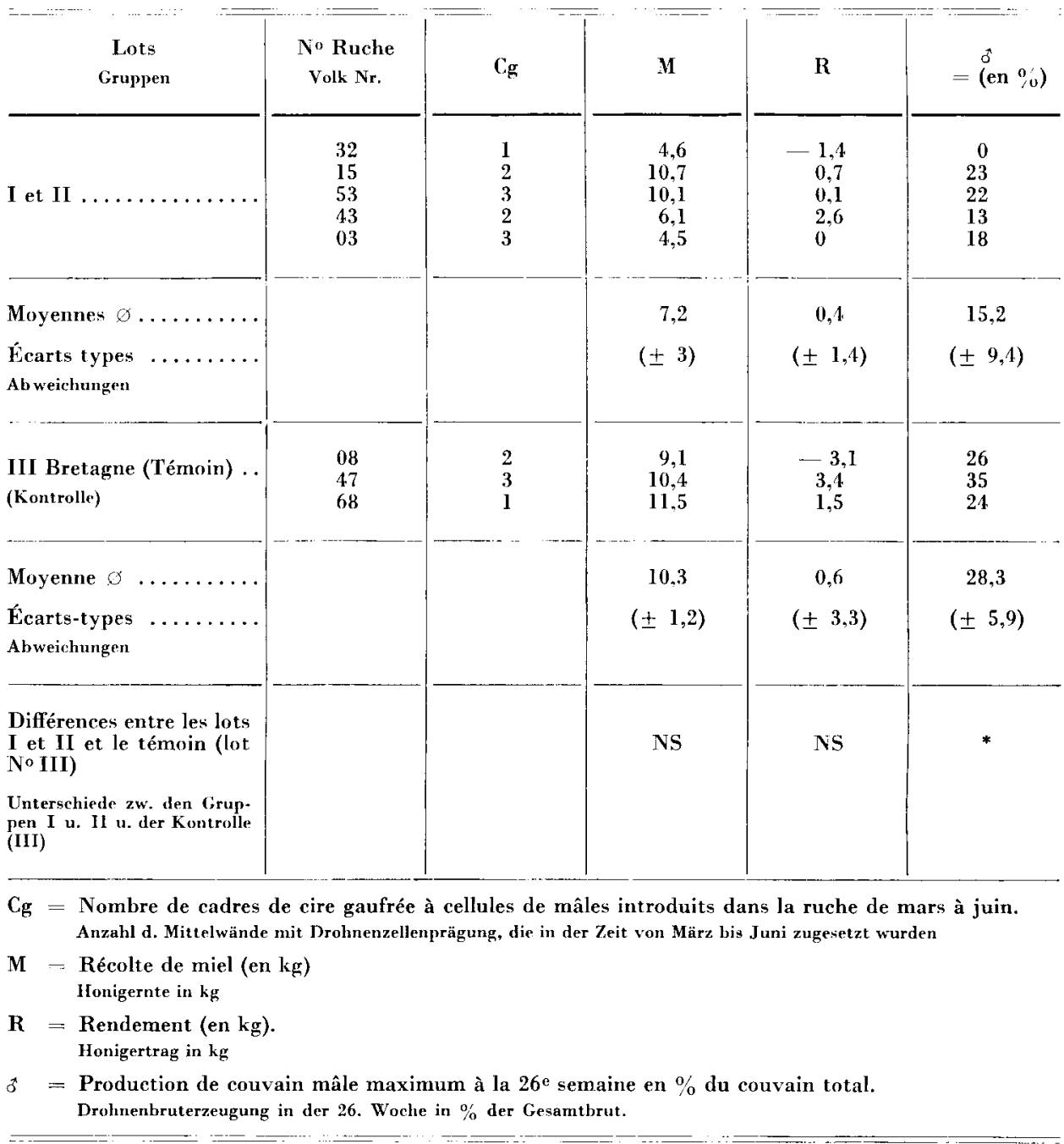

La production de mâles réduite ou nulle ne semble pas être ainsi la conséquence d'une éventuelle carence du comportement de constructions des cellules de mâles par les ouvrières. 


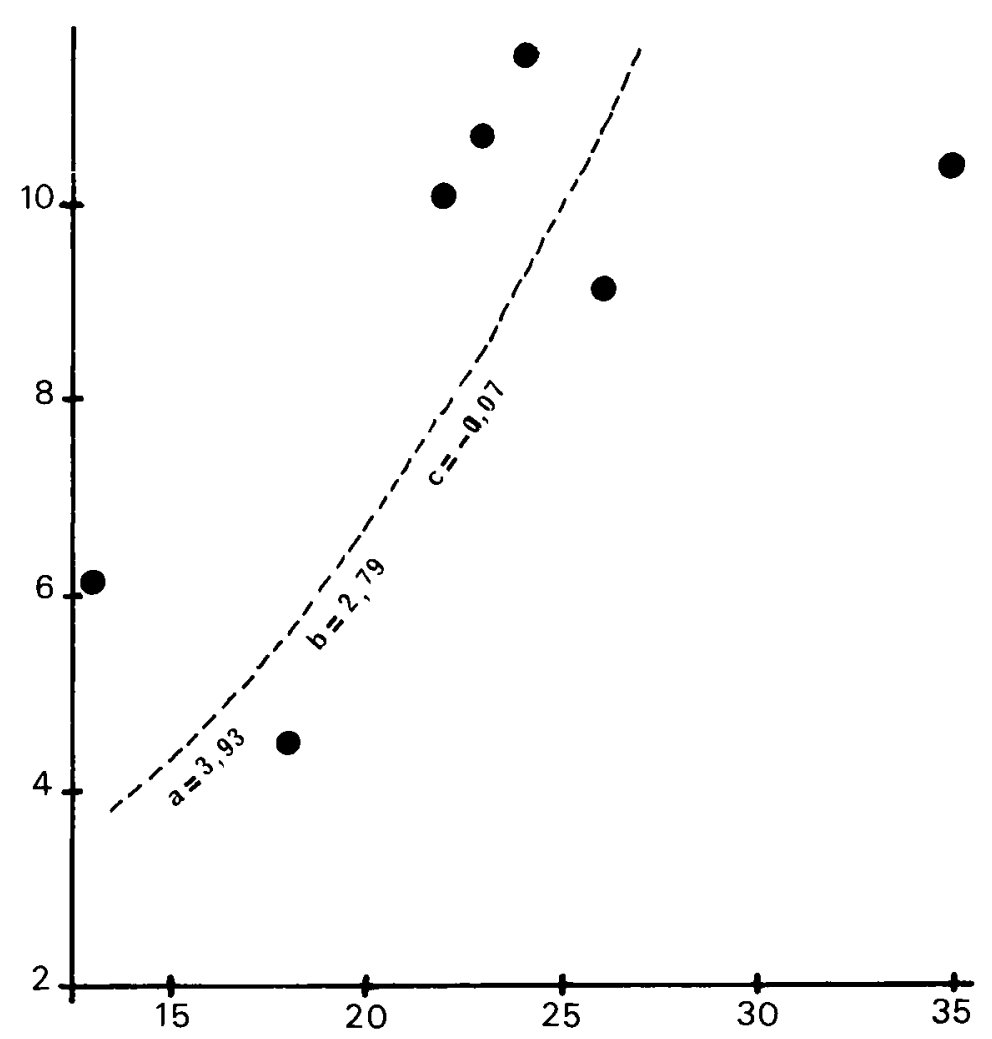

FIG. 3. - Incidence de la surproduction de couvain de mâles sur la rếcolte de miel des colonies d'abeilles des lots II et III à Rennes en 1973.

Ordonnées: Récolte de miel en $\mathrm{kg}$.

Abscisses: : Couvain de mâles en $\%$.

Aвв. 3. - Einfluss der Überproduktion von Drohnenbrul auf den Honigertrag der Bienenvölker der Gruppen II u. III in Rennes 1973 .

Ordinate Honigertrag in $\mathrm{kg}$.

Abszisse … Drohnenbrut in $\%$.

Les résultats obtenus permettent en outre de faire les remarques suivantes :

$1^{\circ}$ Dans l'ensemble, ce sont les colonies du lot II (tableau 5) qui ont eu tendance à donner des surfaces moyennes de constructions cirières en cellules de mâles et en cellules d'ouvrières plus élevées que celles des bretonnes. Ces différences ne sont cependant pas significatives mais l'amplitude de la variation entre ruches apparaît bien plus grande chez les colonies du lot II. En effet, le tableau 5 fait apparaître chez celles-ci des proportions de constructions en cellules de mâles qui peuvent être, selon les ruches, très élevées (jusqu'à $71 \%$ ). Les constructions en cellules de mâles chez les colonies bretonnes apparaissent au contraire plus réduites mais un peu plus homogènes ( $33 \%$ à $51 \%$ ). 
TABL. 5. - Surfaces totales des constructions cirières en cellules d'ouvrières et de mâles sur "cadres témoins " du 15 juin au 19 juillet 1973 des colonies des lots I à III à Rennes.

ТАВ. 5. -- Gesamtfläche von Arbeiterinnen- u. Drohnenbau auf den Baurahmen vom 15.6 bis 19.7.1973 in den Völkern der Gruppen I bis III in Rennes.

\begin{tabular}{|c|c|c|c|c|}
\hline & & & $\begin{array}{l}\text { es des Constru } \\
\text { baute Wabenfläcl }\end{array}$ & \\
\hline Gruppen & Volk Nr. & $\begin{array}{c}\text { Cellules d' } \\
\mathrm{cm}^{2}(\%) \\
\text { Arbeiterinnenzellen }\end{array}$ & $\begin{array}{l}\text { Cellules de } \vec{o} \\
\mathrm{~cm}^{2}(\%) \\
\text { Drohnenzellen }\end{array}$ & $\begin{array}{l}\text { Total } \mathrm{cm}^{2} \\
\text { Gesamt fläche }\end{array}$ \\
\hline $\mathrm{I}^{1} \ldots \ldots \ldots \ldots \ldots \ldots \ldots \ldots \ldots$ & 32 & $905 \quad(47)$ & $1.005 \quad(53)$ & 1.910 \\
\hline II $\ldots \ldots \ldots \ldots$ & $\begin{array}{l}15 \\
53 \\
43 \\
03\end{array}$ & $\begin{array}{rr}567 & (29) \\
833 & (29) \\
1.678 & (95) \\
2.339 & (65)\end{array}$ & $\begin{array}{rr}1.383 & (71) \\
2.018 & (71) \\
85 & (5) \\
1.268 & (35)\end{array}$ & $\begin{array}{l}1.950 \\
2.851 \\
1.763 \\
3.607\end{array}$ \\
\hline $\begin{array}{l}\text { Moyennes } \varnothing \ldots \ldots \ldots \ldots \ldots \ldots \\
\text { Écarts-types } \ldots \ldots \ldots \ldots \ldots \ldots \ldots \\
\text { Abweichungen }\end{array}$ & & $\begin{array}{l}1.354 \quad(53) \\
( \pm 810)\end{array}$ & $\begin{array}{l}1.189(4.7) \\
( \pm 806)\end{array}$ & $\begin{array}{c}2.543 \\
( \pm 854)\end{array}$ \\
\hline $\begin{array}{l}\text { III Bretagne (Témoin) } \\
\text { (Kontrolle) }\end{array}$ & $\begin{array}{l}08 \\
47 \\
68\end{array}$ & $\begin{array}{rr}1.305 & (67) \\
848 & (49) \\
1.184 & (62)\end{array}$ & $\begin{array}{ll}652 & (33) \\
881 & (51) \\
723 & (38)\end{array}$ & $\begin{array}{l}1.957 \\
1.729 \\
1.907\end{array}$ \\
\hline $\begin{array}{l}\text { Moyennes } \varnothing \ldots \ldots \ldots \ldots \ldots \\
\text { Ecarts-types } \ldots \ldots \ldots \ldots \ldots \\
\text { Abweichungen }\end{array}$ & & $\begin{array}{l}1.112(60) \\
( \pm 237)\end{array}$ & $\begin{array}{l}752(40) \\
( \pm 117)\end{array}$ & $\begin{array}{r}1.864 \\
( \pm 120)\end{array}$ \\
\hline $\begin{array}{l}\text { Différences entre lots II et III ... } \\
\text { Unterschiede zw. Gruppe II u. III }\end{array}$ & & NS & NS & NS \\
\hline
\end{tabular}

1. Le lot I n'a pu être constitué qu'avec une seule colonie.

Les surfaces sont données en $\mathrm{cm}^{2}$ sur une seule face du rayon.

Die Flächen sind in $\mathrm{cm}^{2}$ nur auf einer Wabenseite angegeben.

Les chiffres entre parenthèses sont les pourcentages par rapport aux constructions totales. Die Ziffern in Klammern sind die Prozente der Gesamtflächen.

$2^{\circ} \mathrm{Si}$ l'on examine les résultats obtenus par périodes hebdomadaires (tableau 6), on constate d'importantes variations. Les constructions totales sont maximum entre les 15 et 28 juin.

Elles régressent ensuite régulièrement au cours des semaines suivantes jusqu'à la semaine du 12 au 19 juillet; après quoi les constructions ont cessé et n'ont plus repris pendant toute la période estivale. Les constructions totales, pendant la miellée, semblent ainsi s'effectuer selon un rythme régulier et les 
figures 4 et 5 montrent qu'il est sensiblement le même dans les lots de colonies considérées. Statistiquement on ne fait d'ailleurs apparaître aucune différence significative (tableau 6). Par contre, on ne retrouve plus une aussi bonne régularité du rythme des constructions lorsque l'on considère isolément les rayons en cellules de mâles et en cellules d'ouvrières.

\section{2. _- Influence du comportement de destruction du couvain de mâles par les ouvrières.}

Les taux de destruction d'oufs sont identiques (autour de $20 \%$ ) en avril et en mai chez les colonies du lot II et chez les colonies bretonnes (tableau 7). Les taux de destruction larvaires apparaissent par contre différents. Ils sont plus élevés chez les colonies du lot II : $49 \%$ et $28 \%$ contre $8 \%$ pour les bretonnes. Le taux de destruction totale apparaît par conséquent plus élevé chez les colonies du lot II : $69 \%$ et $52 \%$ contre $37 \%$ et $26 \%$ pour les bretonnes.

Les taux de destruction des oufs et des larves en juin et juillet prennent une autre physionomie. Si l'on n'observe pas de destruction d'œufs en juin dans les deux lots de colonies, on observe, par contre, une destruction totale des larves dix jours plus tard en juillet. Mais ceci semble correspondre à une cessation de production de couvain de mâles (fig. 2).

\section{DISCUSSION}

\section{A. - Différents aspects de la variabilité de la production de couvain de mâles}

Les résultats obtenus montrent qu'il y a eu en 1972 et 1973 maintien d'un élevage de mâles nul ou réduit chez les colonies des lots I et II. Ce phénomène peut donc s'observer pendant plusieurs années consécutives et plusieurs générations.

Les colonies présentant le caractère “ mâle réduit » se sont d'ailleurs montrées susceptibles de fournir des surfaces moyennes de couvain total plus élevées que celles des témoins bretons alors qu'elles ont fourni des surfaces moyennes de couvain de mâles plus faibles. La réduction de la production de couvain de mâles, étant donné l'absence de proportionnalité, ne semble donc pas liée à une diminution de la production de couvain total qui aurait pu correspondre à un éventuel affaiblissement des colonies.

Si l'on examine les résultats dans le détail, on remarque que la production de couvain de mâles de ces colonies (lots I et II) se montre sous deux aspects différents :

- l'un est caractérisé par une persistance d'un état de production de mâles généralement pratiquement nulle qui peut se retrouver sur plusieurs années, plusieurs générations (lot I). 
TABL. 6. - Moyennes des surfaces des constructions cirières hebdomadaires sur "cadres témoins " du 15 juin au 19 juillet 1973 des colonies des lots I à III à Rennes.

TAB. 6. - Durchschnitt der wöchentlich gebauten Wabenflächen auf den «Baurahmen » der Völker in den Gruppen I bis III vom 15.6 bis 19.7.1973 in Rennes.

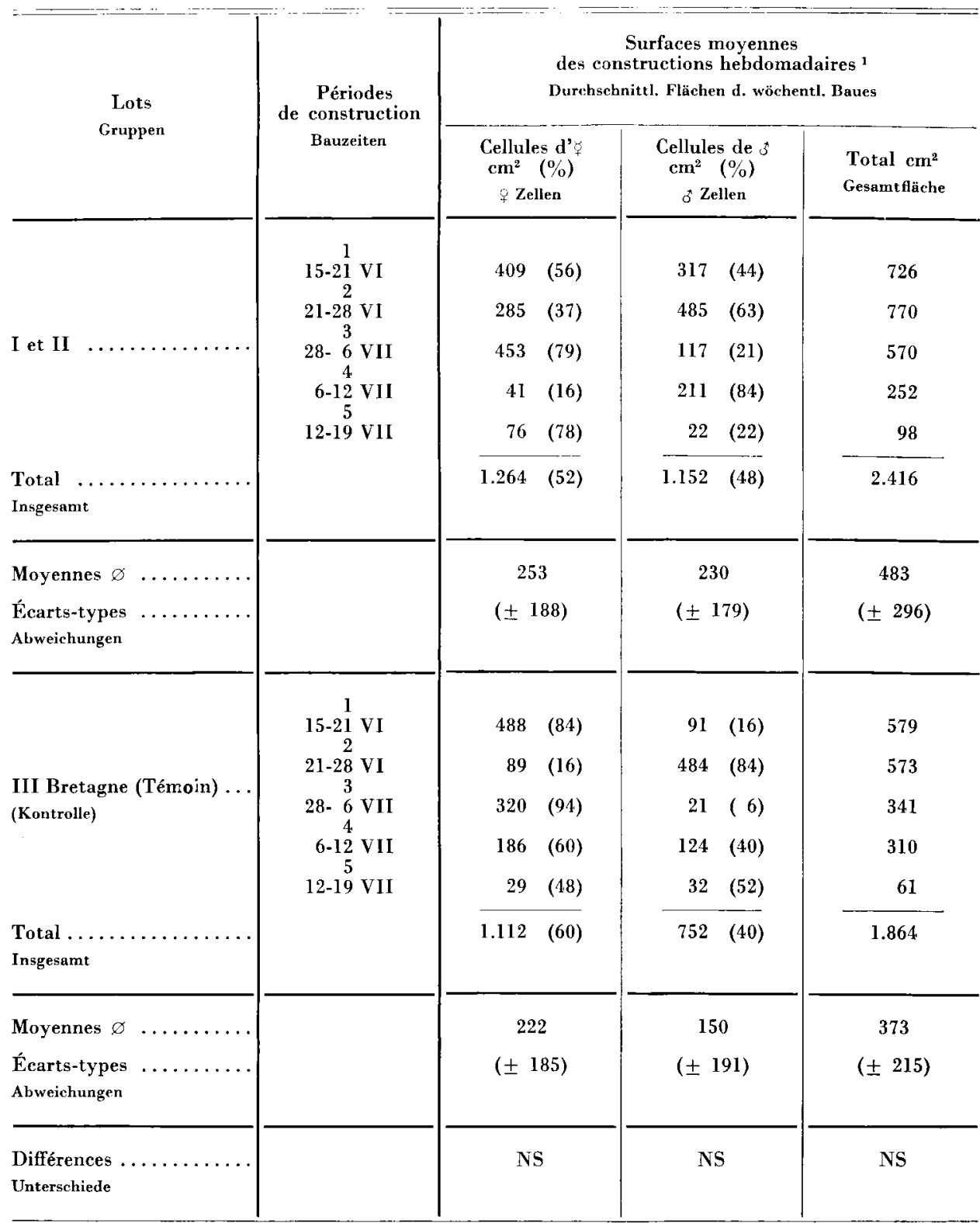

1. Surfaces moyennes de construction d'une seule face du rayon. Durchschnittliche Fläche auf nur einer Wabenseite. 


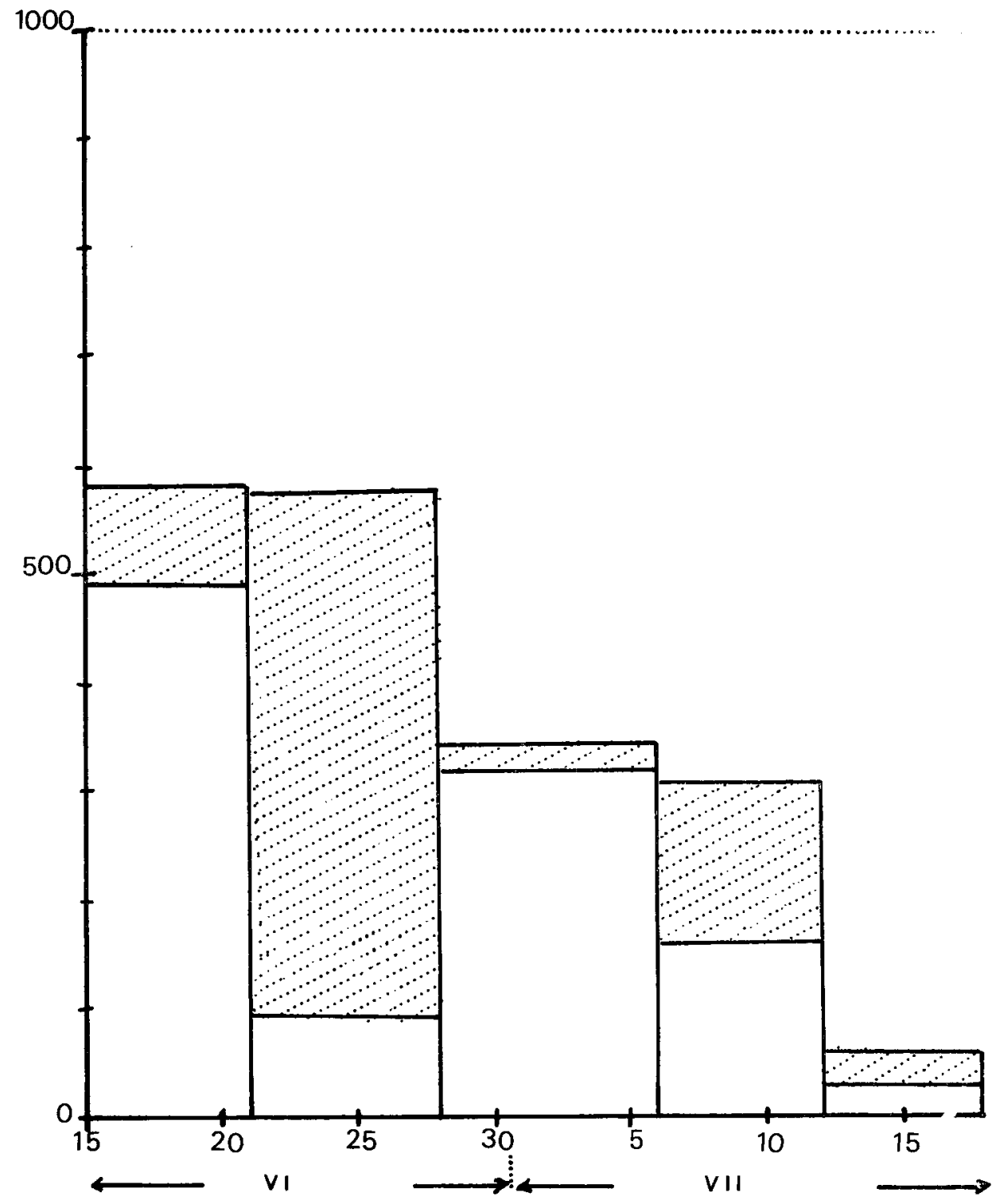

FIG. 4. - Rythme des constructions cirières des colonies d'abeilles des lots I et II du 15 juin au 19 juillet 1973 à Rennes.

Ordonnées: Surfaces en $\mathrm{cm}^{2}$.

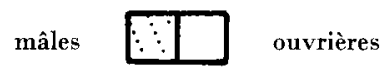

Aвв. 4. - Rhythmus des Wabenbaues bei den Völkern der Gruppen I und II vom 15.6. bis 19.7.1973 in Rennes.

Ordinate $=$ Wabenflächen in $\mathrm{cm}^{2}$.

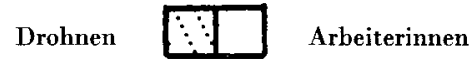




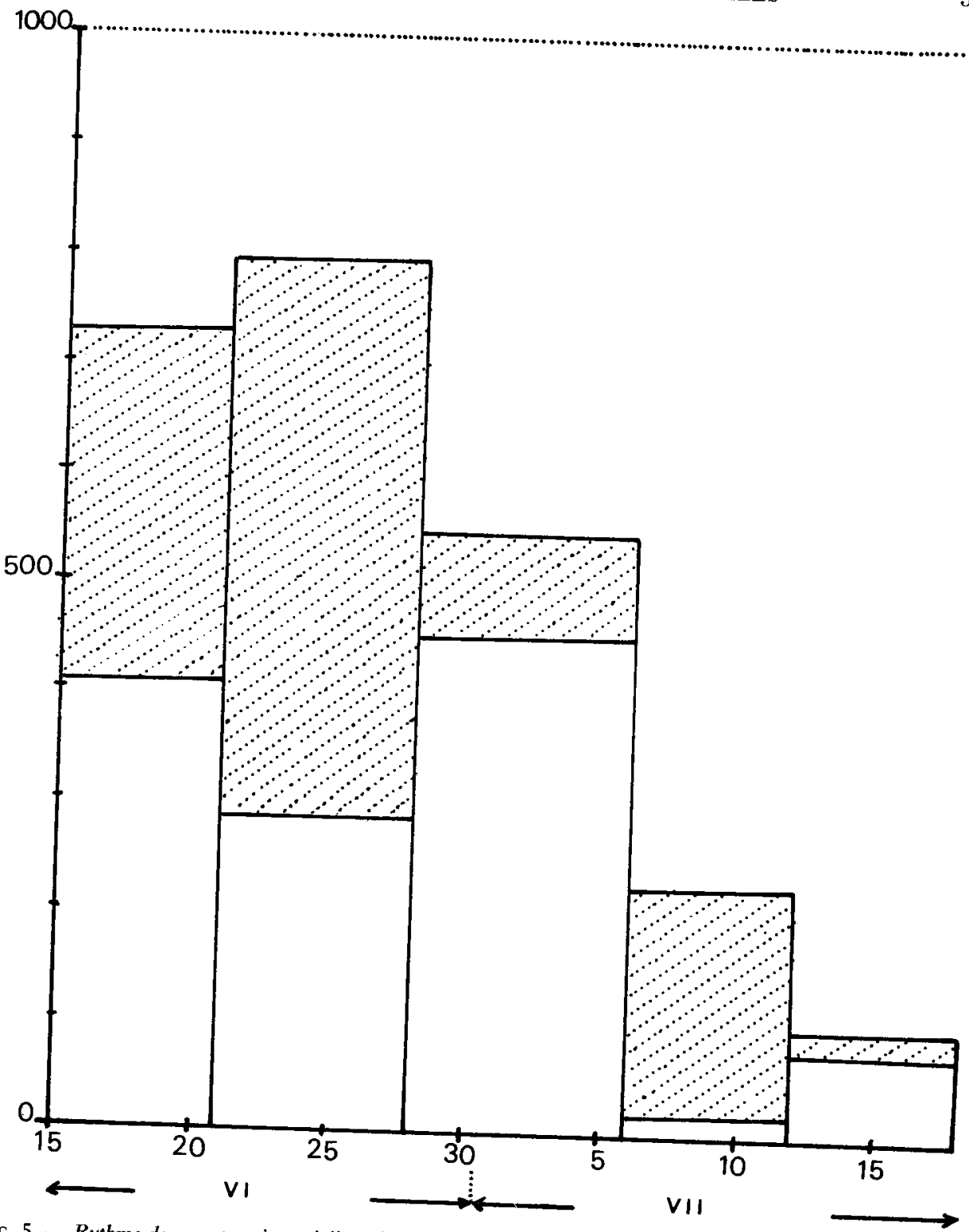

FIG. 5. - Rythme des constructions cirières des colonies d'abeilles bretonnes (lot III) du 15 juin au 19 juillet
1973 a Rennes. Ordonnées : surfaces en $\mathbf{c m}^{2}$.

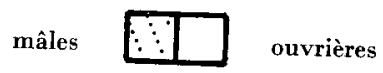

Aвв. 5. Rhythmus des Wabenbaues bei den bretonischen Völkern (Gruppe III) vom 15.6. bis 19.7.1973
in Rennes. Ordinate $=$ Wabenfläche in $\mathrm{cm}^{2}$.

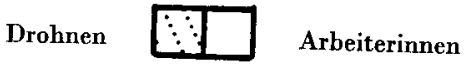


J. MESQUIDA

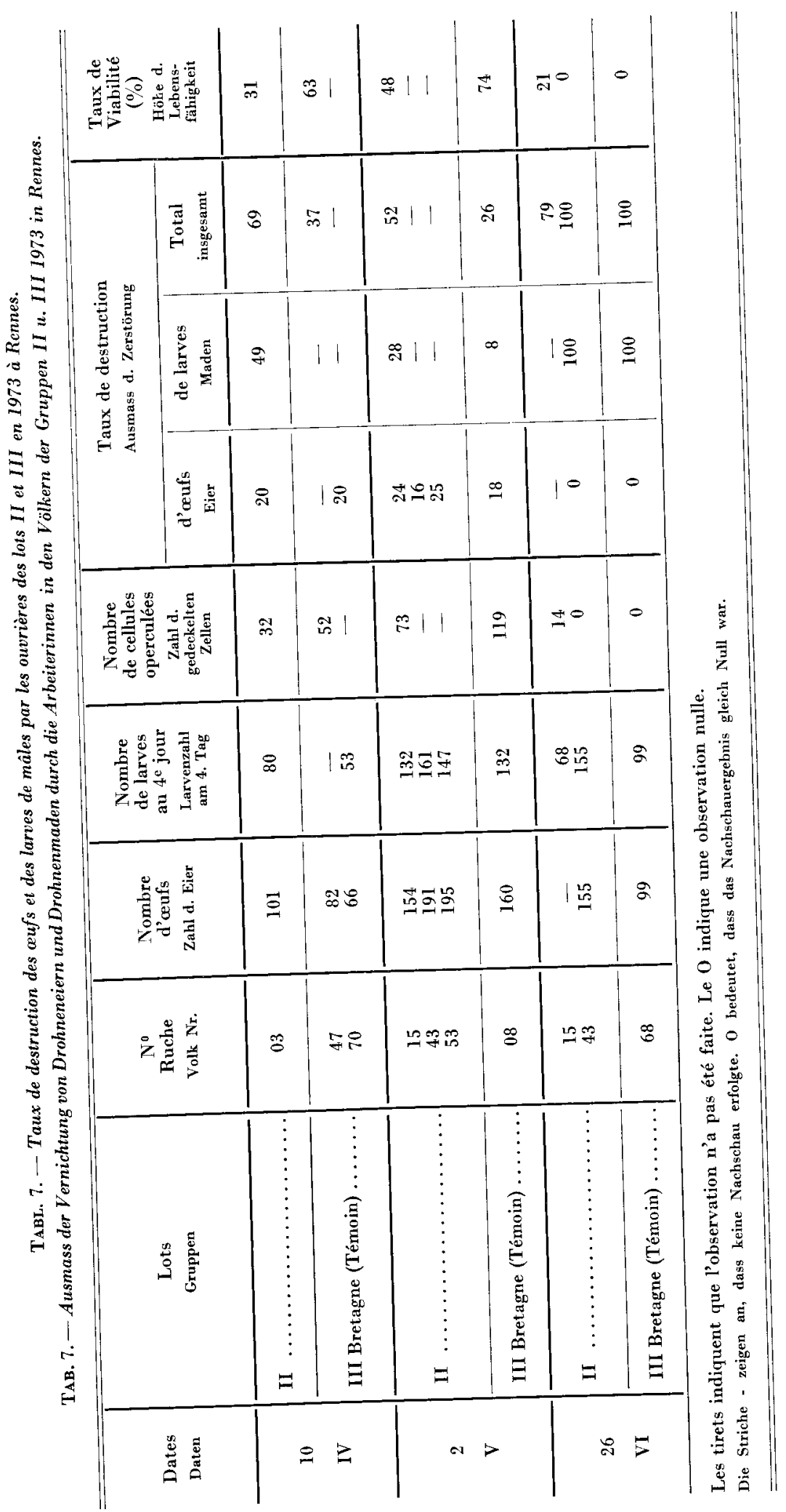


- l'autre est caractérisé par des modifications progressives du phénomène en fonction du renouvellement des reines. La production de couvain de mâles, en constante augmentation d'une génération à l'autre, passe par des états intermédiaires (lot II).

L'un et l'autre aspects mettent en évidence le caractère génétique du phénomène comme nous l'avions déjà signalé en 1972 (Louveaux, Mesquida, Fresnaye) et l'augmentation par étapes intermédiaires de la production de couvain de mâles montre que ce caractère génétique pourrait dépendre de plusieurs gènes. Mais, si ces deux aspects semblent liés au même phénomène, le premier représentant la phase initiale, il y a lieu cependant de faire les remarques suivantes :

Lorsque, dans le premier cas, les générations successives ne parviennent pas rapidement à retrouver une production normale de couvain de mâles, donc à entrer dans la seconde catégorie, une régression de la ponte d'ouvrières et de la population s'ensuit au cours des générations successives, jusqu'à rendre la colonie plus vulnérable à l'hivernage; cela est peut-être à l'origine de leur extinction. Inversement, dans le second cas, les colonies évoluent vers un comportement « normal ».

\section{B. - Essai d'explication du phênomène “élevage de mâles réduit ou nul »}

\section{Influence de l'évaluation de la taille des cellules par les reines.}

Les résultats obtenus permettent de dire que le phénomène ne dépend pas d'une défaillance dans l'évaluation de la taille des cellules par les reines.

Plus nettement que les autres colonies, c'est la colonie No 32 qui fait mieux apparaître ce caractère. En effet, si la reine n'a pas déposé d'œufs de mâles dans ces cellules, ou si elle n'y a pas pondu davantage d'œufs d'ouvrières, c'est qu'elle les a bien évaluées. L'emplacement du cadre à cellules de mâles dans la ruche, entre deux cadres de couvain ouvert d'ouvrières avec présence d'œufs dans chacun d'eux laisse supposer que la reine a dû l'explorer à maintes reprises.

La présence de trois oufs pondus dans les grandes cellules ayant donné naissance à des ouvrières s'explique mal mais n'est pas suffisante pour justifier une conclusion différente de celle que nous venons d'exposer.

2. Influence de l'aptitude à la construction des cellules de mâles.

Le phénomène ne semble pas dépendre non plus du comportement des ouvrières par l'intermédiaire des constructions des cellules de mâles. Comme dans les colonies témoins, des constructions des cellules de mâles ont été en effet obtenues normalement aussi bien sur des cadres de cire gaufrée à cellules de mâles que sur des cadres vides par l'ensemble de ces colonies, y compris la colonie No 32 . 


\section{Influence des soins apportês au couvain par les ouvrières}

Ce facteur ne semble pas entrer en ligne de compte.

La réduction de couvain de mâles ne semble pas relever de sa destruction par les ouvrières. Les résultats obtenus permettent de constater une destruction d'oufs aussi importante dans les colonies du lot II que dans les colonies témoins. Les destructions de larves se produisent également partout mais elles apparaissent moins importantes dans les colonies témoins.

Les destructions systématiques du couvain ouvert de mâles qui se sont produites en juillet ne répondent pas à notre hypothèse. Il s'agit là d'un autre phénomène qui semble lié à l'état physiologique de la colonie. Au moment où il se produit, on observe aussi une diminution très sensible de la ponte, l'arrêt de la construction des cellules, la fin de la miellée, etc. Il est donc en rapport avec le déroulement normal du cycle biologique annuel.

Notons que la méthode d'élevage de mâles poussée au maximum, appliquée en 1973 à l'ensemble des colonies, a permis, avec l'introduction de 1 à 3 cadres de cire gaufrée à cellules de mâles selon les nécessités, d'augmenter notablement la production de couvain de mâles sans nuire à l'élevage des ouvrières d'une part, à la récolte du miel d'autre part, et sans aucun rapport avec la préparation à l'essaimage. Il faut ajouter que l'introduction de cadres de cire gaufrée à cellules de mâles a eu un effet favorable sur la répartition du couvain. La majeure partie, et quelquefois la totalité du couvain de mâles, se trouvait concentrée sur ces cadres alors qu'en leur absence il se trouvait réparti d'une façon très hétérogène dans les angles du bas des cadres de couvain d'ouvrières et sur des surfaces plus grandes des cadres d'ouvrières situées sur les bords du nid.

En conclusion, le caractère génétique du phénomène décrit se confirme. Le fait que la production de couvain de mâles tende à augmenter et à redevenir normale au cours des générations successives, semble prouver l'influence de plusieurs gènes en rapport avec ce caractère.

Le mécanisme du phénomène ne semble pas relever de la reine par l'intermédiaire d'une défaillance de l'évaluation de la taille des cellules, de même qu'il ne semble pas relever des ouvrières par l'intermédiaire des constructions cirières ou de la destruction du couvain. Par contre, l'hypothèse de Gorbakov selon laquelle il s'agirait d'une anomalie du fonctionnement de la spermathèque est à prendre en considération. Une vérification sur un plus grand nombre de colonies présentant le caractère “ élevage sans mâles " serait nécessaire mais la rareté du phénomène en complique singulièrement l'étude. 


\section{ZUSAMMENFASSUNG}

Aus früheren Arbeiten (Louveaux, Mesquida, Fresnaye 1972) ist bekannt, dass es Bienenvölker (Apis mellifica L.) gibt, die nur wenige oder gar keine Drohnen aufziehen.

1972 und 1973 haben wir, um die Untersuchungen über Ursprung und Mechanismus dieses Phänomens in Angriff nehmen zu können, eine Reihe von Beobachtungen durchgeführt, die sich auf mehrere Hypothesen stützten.

Die erste Hypothese befasst sich mit dem Verhalten der Königin, d.h. mit ihrem Unvermögen, die Zellengrösse richtig wahrzunehmen oder mit dem ungenügenden Funktionieren der Spermatheka (Hypothese von GorBakov).

Die zweite und dritte Hypothese betreffen die Arbeiterinnen. Es könnte sich hier entweder um ihre Unfähigkeit, Drohnenzellen zu bauen, handeln oder um die Vernichtung der Drohnenbrut.

Es wurde mit drei Gruppen von Bienenvölkern gearbeitet :

1. Gruppe : ein einziges Bienenvolk, dessen Drohnenaufzucht gleich null ist; bei der Königin handelt es sich um eine Urenkelin der Ursprungskönigin (4. Generation).

2. Gruppe : vier Völker mit verminderter Drohnenaufzucht, deren Königinnen Enkelinnen (3. Generation) oder Urenkelinnen (4. Generation) der ursprünglichen Königin sind.

3. Gruppe : drei oder vier Völker mit Nigra-Königinnen (Apis mellifica mellifica), die zu einer lokalen, bretonischen Population gehören - Kontrollvölker.

Die Beobachtungen erstreckten sich hauptsächlich auf :

- die Drohnenaufzucht bei den Nachkommen der verschiedenen Beobachtungsvölker,

- die Fähigkeit der Königinnen, die Grösse der Wabenzellen genau wahrzunehmen, d.h. systematische Beobachtung der Erzeugung und Entwicklung von Drohnenbrut in den betreffenden Völkern,

— die Fähigkeit der Arbeiterinnen, Drohnenzellen auf Mittelwänden mit Drohnenzellenprägung und in leeren Rähmchen zu bauen,

- die Vernichtung der Drohnenbrut durch die Arbeiterinnen; d.h. Eier, Maden und Puppen des gleichen Geleges im gleichen, vorher bestimmten Wabenstück nach und nach und in verschiedenen Entwicklungsstadien der Brut zu zählen.

\section{Ergebnisse}

Das Bienenvolk der ersten Gruppe hat auch 1972 und 1973 keine Drohnen gezogen; die Völker der 2. Gruppe neigten zu normaler Drohnenaufzucht (Tab. 1 u. 2; Abb. 1 u. 2). Die Honigerträge wichen nicht von der Norm ab (Tab. 3 u. 4), selbst 1973 nicht, wo eine Überproduktion von Drohnen experimentell ausgelöst worden war (Tab. 4; Abb. 3).

Aus allen Eiern, die in den Drohnenzellen der Völker der Gruppe 2 abgelegt wurden, entstanden ganz normale Drohnen, genau wie in den Kontrollvölkern. Nur die Königin des Volkes in Gruppe 1 bestiftete die grossen Zellen nicht, mit Ausnahme von drei Zellen. Aus diesen drei Eiern entstanden aber Arbeiterinnen.

Die Arbeiterinnen aller beobachteten Völker haben Drohnenzellen und Drohnenwaben gebaut. Während der Zeit der Honigtracht bauten sie in regelmässigem Rhythmus fast ebensoviel Drohnen-wie Arbeiterinnenzellen (Tab. 5 u. 6).

Das Ausmass der Zerstörung von Drohnenbrut durch Arbeiterinnen in den Völkern der 2. Gruppe entsprach im Eistadium dem der Kontrollvölker, war jedoch im Madenstadium viel grösser (Tab. 2).

Es wurde festgestellt, dass die Eigensehaft “ keine oder wenig Drohnenaufzucht » sich unter zwei Aspekten darstellt :

— der eine ist charakterisiert durch die Beständigkeit dieser Eigenschaft bis zur 4. Generation, 
- der andere ist charakterisiert durch fortschreitende Veränderungen der Drohnenaufzucht in aufeinanderfolgenden Generationen.

Die anfänglich sehr geringe Drohnenaufzucht zeigt die Tendenz, nach mehreren Generationen « normal » zu sein. Die Erbeigenschaft dieses Phänomens, das dem Einfluss mehrerer Gene unterworfen zu sein scheint, dürfte mehr als fraglich erscheinen.

Es wurde ferner festgestellt, dass dies Phänomen nicht auf einer fehlerhaften Einschätzung der Zellengrösse durch die Königin beruht, ebensowenig wie auf einem mangelhaften Wabenbau oder der Zerstörung der Drohnenbrut durch die Arbeiterinnen.

Es scheint, dass die Hypothese von Gokbakov diesem Phänomen eher entspricht, und dass es sich somit um die Funktion der Spermatheka handeln dürfte. Um aber zu einem gültigen Schluss zu gelangen, wäre zur Bestätigung dieser Hypothese die Beobachtung einer viel grösseren Zahl von Völkern notwendig.

\section{RÉFÉRENCES BIBLIOGRAPHIQUES}

Allev M. D., 1958. - Drone brood in honey bee colonies. J. écon. Entomol., 51, 46-48.

Allen M. D., 1963. - Drone production in honey bee colonies. Nature, 199, ( $\left.\mathrm{n}^{\circ} 4.895\right), 789-790$.

ALLen M. D., 1965. - The effect of a plentiful supply of drone comb on colonies of honey bees. J. apic. Res., 4, 109-118.

Brisset A., 1946. - Étude de la méthode du Dr PAschke. In : l'abeille et son travail, 2, 67-80. Vautrain, Paris.

Dagnelie P., 1969. - Théorie et méthodes statistiques, 1, 1-378. Duculot, Gembloux.

Dagnelie P., 1970. - Théorie et méthodes statistiques, 2, 1-451. Duculot, Gembloux.

Drescher W., 1968. - Élevage et conservation des reines et des mâles. Ann. Abeille, 11, 255-266.

Fresnaye J., 1962. - Un appareil pour le calcul rapide des surfaces de couvain dans les ruches. Ann. Abeille, 5, 145-153.

Gorbakov W. K., 1961. - Colonies sans mâles - apiculture future (en polonais). Pszczelarstwo, 12, 19-21, (in Apicultural abstracts, 16, 145).

Herold E., 1960. — Grundzüge der Bienenhaltung. In : Biene und Bienenzucht, 244-271. Ehrenwirth Verlag, München.

Koeniger N., 1970. - Über die Fähigkeit der Bienenkönigin, zwischen Arbeiterinnen- und Drohnenzellen zu unterscheiden. Apidologie, 1, 115-142.

Lavie P., 1968. - L'étude expérimentale de la conduite des ruches. In : Traité de Biologie de l'A beille, 4, 54-162. Masson, Paris.

Louveaux J., Mesquida J., Fresnaye J., 1972. - Observations sur la variabilité de la production de couvain de mâles dans les colonies d'abeilles (Apis mellifica L.). Apidologie, $3,291-307$.

Mavl V., 1972. - Programme d'élevage avec des allèles sexuels déterminés. C.R. du Symposium international sur le contrôle de l'accouplement et sélection chez l'abeille mellifère, 75-79. Apimondia, Bucarest.

Michailov A. S., 1927. - (In Gorbakov W. K., 1961 - Apicultural Abstracts, 16, 145.)

Paschke J., 1939. - Der Baurahmenimker. Leipzig (In Brisset et Herold).

WeIss K., 1962. - Untersuchungen über die Drohnenerzeugung im Bienenvolk. Arch. $f$. Bienenkd., 39, 1-7.

Weiss K., 1969. - Drohnen im Natur-und Pflegevolk. Allg. Dtsch. Imkerztg., no 3, 100-105; $n^{\circ} 5,131-135 ; n^{\circ} 6,164-166$.

Wочке J., 1963. - What happens to diploïd drone larvae in a honeybee colony. J. apic. Res., 2, 73-75.

Woyke J., 1965. -- Les faux bourdons diploïdes. C.R. du XXe Cong. Intern. d'apic. 163-167. Apimondia, Bucarest.

Woyкe J., 1972. - Les allèles sexuels et la fécondation contrôlée. C.R. du Symposium international sur le contrôle de l'accouplement et sélection chez l'abeille mellifère. 69-74. Apimondia, Bucarest. 